\title{
Improving quality of life after spinal cord injury in India with telehealth
}

\author{
Nishu Tyagi ${ }^{1} \cdot$ Shakti Amar Goel ${ }^{2}$ Marcalee Alexander ${ }^{3,4}$
}

Received: 27 February 2019 / Revised: 26 June 2019 / Accepted: 8 July 2019

(C) International Spinal Cord Society 2019

\begin{abstract}
Introduction Despite adequate inpatient rehabilitation, a number of spinal cord injury (SCI) individuals suffer from difficulties at home and in their local environments. This is mainly prevalent in low-middle-income countries (LMIC) due to a lack of qualified personal caregivers. This issue could be addressed with the help of telehealth technology, which may be used in LMICs without economic concerns.

Case presentations A 44-year-old male with C3 AIS C SCI and a 35-year-old female with T12 AIS A SCI were discharged after successful rehabilitation from a tertiary care spinal center. The patients demonstrated gradual loss in their independence, which was evident by monitoring their home activities biweekly for 4 weeks via a combination of telephone calls, live video chat, and WhatsApp. Subsequently after 4 weeks of consistent guidance, pre-post scores after teletherapy were analyzed for the self-care and mobility subcomponents of the self-reported SCIM III.

Discussion After consistent supervised guidance via telehealth, self-care scores improved in the C3 AIS C case from 3 to 15 and in the T12 AIS A case from 4 to 15 , while mobility scores respectively improved from 14 to 27 and 4 to 16 . Identification of individual competencies, performance, and capacity in activities of daily living and participation, selfassessment, caregiver training, and home integration contributed toward successful community integration. This case series documents the benefits of using telehealth and home goal planning in the aftercare of SCI individuals, in order to improve quality of life in their local environment.
\end{abstract}

\section{Introduction}

The goal of rehabilitation is to restore the abilities of persons with SCIs to their ideal level of functioning and

Supplementary information The online version of this article (https:// doi.org/10.1038/s41394-019-0212-x) contains supplementary material, which is available to authorized users.

$\square$ Nishu Tyagi

nishutyagi08@gmail.com

1 Department of Rehabilitation and Telehealth, Indian Spinal Injuries Centre, Delhi, India

2 Department of Spine and Research, Indian Spinal Injuries Centre, Delhi, India

3 University of Alabama at Birmingham, School of Medicine, Department of Physical Medicine and Rehabilitation, Birmingham, USA

4 Telehealth Sexuality Clinic, Spaulding Rehabilitation Hospital, Boston, MA, USA enhance maximum independence in the home and community. Achieving maximal independence in activities of daily of living (ADL) and fulfilling social roles are strongly related to health and well-being of persons with SCIs [1]. Furthermore, it has been documented that participation in social activities leads to a better quality of life (QOL) [2,3]. In order to achieve these goals, continuity of care after discharge from rehabilitation is paramount $[4,5]$. However, achieving continuity of care is often difficult due to geographic distances, lack of transportation, and lack of funds. One means of conquering these barriers to continuity of care may be through the use of telehealth. Therefore, in this case series, we demonstrate the ability to provide supervision and guidance to two individuals with SCIs through the use of telehealth. Furthermore, we highlight the factors which may contribute toward successful community inclusion after SCI in low-middle-income countries (LMIC). 


\section{Case presentations}

"Case 1": A 44-year-old male sustained a C3-C5 AIS C $\mathrm{SCI}$ in a road traffic accident and presented to the Indian Spinal Injuries Center (ISIC) 8 months later for rehabilitation, complaining of a difficulty in using his left hand and the inability to stand. Prior to admission, he had undergone cervical spine surgery at another facility, where he was taught a few exercises and discharged to home without proper rehabilitation. Prior to injury his medical history was insignificant. Manual muscle test (right/left) at the time of admission revealed respective strength in the right and left extremities as $3 / 0$ for elbow flexion, $3 / 0$ for wrist extension, $3 / 0$ for elbow extension, $3 / 0$ for finger flexion, $3 / 0$ for finger abduction, $3 / 3$ for hip flexion, $3 / 3$ for knee extension, $3 / 3$ for ankle dorsiflexion, $3 / 3$ for great toe extension, and $4 / 3$ for ankle plantar flexion. Bladder and bowel function were intact, as determined by clinical examination of perianal sensation and voluntary anal contraction. This was confirmed by ultrasonography (full bladder and post-void residual volume). Uroflowmetry test was also conducted to confirm the same. Though perianal sensation and voluntary anal contraction were present, it was difficult for him to use the washroom independently. Functionally, he reported an inability to use his left hand in routine activities and dependence on caregivers for self-care and basic mobility activities like bathing, dressing, grooming, turning, rolling, and getting in and out of bed, and required full assistance to go to the bathroom. He was pushed in a wheelchair or walked short distances at home, with the assistance of his wife and son without any assistive device. Prior to admission, exercises for elbow flexors, shoulder abductors for the left upper limb, and assisted standing were performed in conjunction with his caregivers without any professional supervision.

The patient had a 1.5-month stay at the ISIC working on improving his functional skills and regaining independence. At the time of discharge, manual muscle strength in the right and left extremities (right/left) was $4 / 2$ for elbow flexion, $4 / 2$ for wrist extension, $3 / 1$ for elbow extension, $4 / 1$ for finger flexion, and $4 / 0$ for finger abduction. Bilateral lower-extremity strength was 4 throughout. Functionally, at the time of discharge, he was independent with an adaptive device for eating and drinking; washing the upper body and head, and upper-body dressing. He required minimal assistance in grooming, washing, and dressing the lower body, and minimal assistance with environmental modifications for bladder and bowel care. For mobility, he was independent in turning, was not using the wheelchair anymore, was independent in sit-to-stand, able to walk indoors, and go up and down the stairs independently with supervision of a therapist. He was prescribed a walker to use with both hands with the assistance of gloves, universal cuff, writing aid, low-cost parallel bar, and cycling device. Upon discharge, he returned to his village about $142 \mathrm{~km}$ from the spine center. At 4 weeks post discharge, he had regressed to being dependent in grooming, washing and dressing his lower body, needed assistance to turn the upper body for transfers and toileting activities, needed assistance with walking and going sit-to-stand, and was unable to climb the stairs, at least, in part because of fear.

"Case 2": A 35-year-old female sustained T12 AIS A paraplegia in a road traffic accident $\sim 1080 \mathrm{~km}$ from ISIC. She underwent decompression and fixation of the spine at an outside facility and was discharged home. She came to ISIC 5 months later for SCI-related expert advice and rehabilitation. At the time of admission, she was dependent for ADL, specifically having issues with balance for dressing, wheelchair skills, and transfers. She had two children and was worried about the impact of her SCI on parenting. She remained in rehabilitation for 2 months. At discharge, she was independent in washing the upper body, head, and lower body; independent in simple upper- and lower-body dressing, grooming, and instrumental ADL, including preparation of meals and simulated mopping. She was independent in turning the upper and lower body, sitting up in bed, and car and bed transfers; independent in mobility at a wheelchair level; independent in intermittent catheterization and performing her bowel program, but needed assistance for commode transfers. Some outdoor visits were performed during rehabilitation to ensure that she could be independent in community situations, like auto/tuk-tuk/three-wheeler transfer (image 1). She was discharged to home, $1083 \mathrm{~km}$ away from the rehabilitation hospital. At 4 weeks after discharge, she regressed to being dependent in washing the upper body, head, and lower body; dependent in dressing the upper and lower body; dependent in combing her long hair due to fear of losing balance and falling from the wheelchair; required assistance for turning and sitting up; required assistance for bed-to-wheelchair transfers; was dependent for car and commode transfers; dependent for catheterizations and had not participated in any outdoor activities.

\section{Our intervention}

For both patients, at the time of discharge an interview was conducted (Table1) to determine specific follow-up needs. Personalized home exercises were prescribed. Mutual, achievable goals were developed and both individuals were asked to send videos or small clips of their daily activities via email or text or WhatsApp which include trained functional skills and exercises prescribed at the hospital twice a week for a month. Due to poor connectivity, individual 1 frequently provided still photos of his ADLs, including assisted feeding and brushing (video 2), assisted turning (video 1), and bilateral arm exercises of shoulder 
Table 1 Interview questions asked from SCI individuals at the time of discharge

\begin{tabular}{ll}
\hline S.No & Questions \\
\hline 1 & Marital status before injury \\
2 & Education status before injury \\
3. & Occupation status at the time of injury \\
4. & Total members in the family \\
5. & Totally monthly income of the family (INR) \\
6. & Internet facility available with patient and caregiver \\
7. & Availability of spine speciality services at patient's local place \\
8. & Availability of rehabilitation services at patient's place \\
\hline
\end{tabular}

flexors (video 7). He was able to send videos of his assisted walking with the help of his son (videos 3-5). Individual 2 sent videos of her activities including propelling the wheelchair indoor, independent transfers (bed to wheelchair, wheelchair to bed, and wheelchair to car), and exercise of hip flexors, abductors, and ankle dorsiflexors (videos $8,9)$. She also sent pictures of her standing posture. In addition, the monitoring team questioned the individuals and caregivers weekly about any hindrances faced while practicing functional skills or exercises at home.

To document the functional status of the individuals, we also used the Spinal Cord Independence Measure (SCIM). Pre-scores on self-care and mobility components of selfreported SCIM III [6] were obtained at the time of discharge. Post-SCIM scores I were recorded 4 weeks after discharge in both individuals. Both individuals had a decline in their ADL and mobility skills during the first 4 weeks of post discharge. Based upon SCIM III, self-care scores decreased $(45-15 \% ; 90-20 \%)$ and mobility scores decreased $(60-35 \% ; 42.5-12.5 \%)$ during the initial 4 weeks post discharge.

After the initial 4 weeks at home, based on the telefollow-up, the care team provided new video clips of customized exercises and guidance to individuals 1 and 2 five days a week for an additional 4 weeks. Subsequently consistent customized guidance was carried out in the form of live video chat, consistent telephonic reminders, and videobased training solutions. Telephonic reminders were performed biweekly and video-based training solutions were individualized as per the patient's requirement, demonstrating the correct techniques of functional activities which include transfers, walking pattern, bed-mobility activities, etc. The videos were made by therapists who had the clear understanding of SCI and related problems to provide clear demonstration of the techniques. During the second 4 weeks post discharge, both individuals had consistent monitoring of exercises, functional activities, and timely instructions to the caregivers through live video chats, video training, and telephonic/WhatsApp reminders. When providing the
Table 2 Pre- and post-SCIM III scores reported by SCI individuals after teletherapy

\begin{tabular}{|c|c|c|c|c|c|c|}
\hline \multirow[t]{2}{*}{ SCIM III } & \multicolumn{3}{|c|}{ Case 1} & \multicolumn{3}{|c|}{ Case 2} \\
\hline & Pre & Post I & Post II & Pre & Post I & Post II \\
\hline \multicolumn{7}{|l|}{ Self-care component } \\
\hline Eating and drinking & 2 & 1 & 3 & 3 & 3 & 3 \\
\hline $\begin{array}{l}\text { Washing the upper body } \\
\text { and head }\end{array}$ & 2 & 1 & 2 & 3 & 0 & 2 \\
\hline Washing your lower body & 1 & 0 & 2 & 3 & 0 & 2 \\
\hline Dressing your upper body & 2 & 1 & 3 & 3 & 0 & 3 \\
\hline Dressing your lower body & 1 & 0 & 2 & 3 & 0 & 2 \\
\hline Grooming & 1 & 0 & 3 & 3 & 1 & 3 \\
\hline Total self-care score (20) & 9 & 3 & 15 & 18 & 4 & 15 \\
\hline \multicolumn{7}{|l|}{ Mobility component } \\
\hline Mobility activities & 4 & 2 & 4 & 6 & 2 & 6 \\
\hline Bed to wheelchair & 2 & 1 & 2 & 2 & 1 & 2 \\
\hline Wheelchair to tub/toilet & 1 & 0 & 1 & 1 & 0 & 1 \\
\hline $\begin{array}{l}\text { Moving around/walking } \\
\text { indoors }\end{array}$ & 8 & 3 & 8 & 2 & 2 & 2 \\
\hline $\begin{array}{l}\text { Moving around/walking } \\
\text { moderate distances }\end{array}$ & 3 & 3 & 6 & 2 & 0 & 2 \\
\hline $\begin{array}{l}\text { Moving around/walking } \\
\text { outdoor distances }\end{array}$ & 3 & 3 & 3 & 2 & 0 & 2 \\
\hline $\begin{array}{l}\text { Going up and down } \\
\text { the stairs }\end{array}$ & 1 & 0 & 1 & 0 & 0 & 0 \\
\hline $\begin{array}{l}\text { Transfers from a } \\
\text { wheelchair into a car }\end{array}$ & 2 & 2 & 2 & 2 & 0 & 1 \\
\hline $\begin{array}{l}\text { Transfer from floor to a } \\
\text { wheelchair }\end{array}$ & 0 & 0 & 0 & 0 & 0 & 0 \\
\hline Total mobility score (40) & 24 & 14 & 27 & 17 & 5 & 16 \\
\hline
\end{tabular}

Pre: at discharge, Post I: after 4 weeks at home, Post II: after telerehabilitation program of 4 weeks

video-based exercises, the team ensured that subtitles in the local language along with the frequency of repetitions were maintained for ease of understanding. After the consistent support and guidance during these 4 weeks, post-SCIM scores II were noted for both the individuals. No other therapy was provided to either individual. Both individuals completed all sessions with a compliance rate of $100 \%$. Self-care and mobility scores exhibited a notable improvement after 4 weeks of consistent guidance from post-score I to post-score II. For individual 1, self-care and mobility scores increased from 15 to $75 \%$ and 35 to $67.5 \%$. For individual 2 , self-care and mobility scores increased from 20 to $75 \%$ and 12.5 to $40 \%$ (Table 2).

\section{Discussion}

This is the first report to document the benefits of telehealth follow-up monitoring and therapy for persons with SCIs in 
India to assist in the transition from the hospital to the community. These cases demonstrate that this type of program may positively affect QOL and functional status of persons with SCI living in the community.

The reasons behind the decline in independence of case 1 were likely the lack of a proper environment for practicing achieved skills (video 1, images 2-4), lack of knowledge in handling routine home tasks, frequent change of a caregiver that made him feel demotivated and led to less participation, and poor self-compliance. In case 2 the individual reported she was being pampered and others insisted on caring for her at home. Hence, she did not practice any self-care and mobility activities, except eating, drinking, brushing, basic bed mobility, and washing the face and hands. These changes led to a major decline in functional status.

The team also sent the highlighted images of the wrong movements captured in the videos sent by the patients and provided the correct instructions for movement via video which led to a $100 \%$ compliance and response rate from the patients and caregivers. Post II scores of both the individuals then improved to near prescores. Therefore, we believe that the 4 weeks of guidance/follow-up care support during the post-discharge acute period was beneficial to allow the individuals to regain their achieved skills.

In our cases, the barriers included an inaccessible environment, equipment issues, and attitudinal barriers. This is consistent with literature where common categories of self-reported barriers were mobility and equipment issues (23\%), lack of environmental and home assistance (20\%), and insurance issues (18\%) [7]. We believe that planning of post-discharge goals is equally important as in-hospital goal planning. This is also evident in literature pertaining to discharge planning and post-discharge care. A metaanalysis showed that comprehensive discharge planning plus post-discharge support for older patients with congestive heart failure reduced readmission rates significantly and improved health outcomes, such as survival and QOL without increasing costs [8]. Another study reported that a structured, systematic, multidisciplinary discharge care plan [9], tailored to the individual patient [10] and coordinated system of hospital discharge system, is required to facilitate the discharge process to ensure a smooth patient transition from the hospital to the community and improve patient health outcomes both clinically and socially [11]. One study conducted in persons with SCIs stressed that timely coordination and collaboration between the patient and his/her family, the rehabilitation center and the community was of great importance to the individuals with SCIs [12]. Hence, the present study strongly suggests that post-discharge goal planning must be considered in the transition process from hospital to home. Furthermore, monitoring of planned home goals through telehealth may lead to increased independence and a successful transition from home integration to community integration.

Both our cases stressed the importance and need of a standardized approach to post-discharge care to assist persons with SCIs and therapists to plan for community reintegration. Five major aspects were identified and reported by the individuals that may contribute to achieve successful community integration after SCI in LMIC. First, identification of specific trainable competencies of an individual i.e., realistic home goal plans and assessment of current living environment. Second, evaluation of performance and capacity toward activities of daily living and participation separately, which may support good adherence and compliance toward participation in the community. In particular, at discharge, all caregivers should be informed about environmental factors, which may act as barriers or facilitators, individuals' capacity, performance, and participation, so that they can continue to practice their trained skills at home. For instance, individual one could supervise his son in farming activities and individual two could perform household activities independently and drive on her own to pick up her children from the school. Third, an opportunity to find performance gaps through self-assessment may be achieved by encouraging the participants to send self-care and mobility skills videos to the specialised centre for further guidance and support. Fourth, it is imperative that a primary caregiver is identified and trained about the amount of assistance needed for the SCI individual at home and community. Fifth, it is also important to encourage the SCI consumers toward identification and maintenance of their individual role as an active participant in the home environment which may assist in community participation. Both individuals in our report noted that greater home participation lead to better confidence in the community. Hsiang$\mathrm{Yu}$ Yang et al. have highlighted that performance challenges at home are positively correlated to less community participation [13]. Furthermore, it has been seen that in SCI individuals, benefits outweigh the required efforts when they have a good reason to participate [14].

In addition to a lack of community participation, in India, it has also been observed that many SCI individuals do not understand the need to act or assist in home decisions and management. The individuals in our case series reported participation in their home environment, which gave them a sense of accomplishment, motivation, and confidence. This resonates with a recent qualitative report on SCI that stated $56 \%$ (over half) of 18 veterans with SCI emphasized the positive effects of participation in sports, faith-based activities, being outdoors, and managing business or householdrelated responsibilities, may improve social and community participation [15]. Hence, we believe that active follow-up may facilitate persons with SCI to achieve greater 
community integration. We also believe using telehealth is an opportune way to assist patients in adjusting to their home environment and maintaining their functional potential. Telehealth saves travel time for patients, money related to the cost of travel, and is an environmentally sound way to deliver healthcare [16]. In this study, the amount spent on an individual for customized support, using common available technology (Internet connection and telephonic calls), was around US \$11.80 per month. We believe that further utilization of low-cost telehealth methods, along with continuous monitoring of persons with SCI, is warranted in lowincome communities and communities with geographical difficulties, etc., to promote increased function at home. However, it is imperative to understand that there are issues that need to be answered on legal considerations concerning telehealth services in different countries and different health systems. Further research is warranted to determine the duration needed of telehealth-telerehabilitation for patients with SCI, and the efficacy in promoting increased community integration and the ultimate improved QOL for persons with SCIs.

Acknowledgements We would like to thank both the individuals and their caregivers for their participation. The authors would like to give special thanks to Dr H.S. Chhabra and Dr Chitra Kataria for the constant support.

\section{Compliance with ethical standards}

Conflict of interest NT, SAG, and MA declare no conflicts of interest; however, MA is currently developing plans to develop a nonprofit organization, Telerehabilitation International, in which she plans to recruit volunteer physiatrists to provide telehealth to persons with neurologic disabilities.

Publisher's note: Springer Nature remains neutral with regard to jurisdictional claims in published maps and institutional affiliations.

\section{References}

1. Schonherr MC, Groothoff JW, Mulder GA, Eisma WH. Participation and satisfaction after spinal cord injury: results of a vocational and leisure outcome study. Spinal Cord. 2005;43:241-8.
2. Cox RJ, Amsters DI, Pershouse KJ. The need for a multidisciplinary outreach service for people with spinal cord injury living in the community. Clin Rehabil. 2001;15:600-6.

3. Tzanos IA, Mitsiokapa E, Megaloikonomos PD, Igoumenou VG, Panagopoulos GN, Papathanasiou J, et al. Social reintegration and quality of life after spinal cord injury: the Greek paradigm. J Biomed. 2016;1:36-43.

4. Ernst JL, Thomas LM, Hahnstadt WA, Piskule AA. The selfidentified long-term care needs of persons with SCI. SCI Psychosoc Process. 1998;1:127-32.

5. Rawl SM, Easton KL, Kwiatkowski S, Zemen D. Burczyk effectiveness of a nurse-managed follow-up program for rehabilitation patients after discharge. Rehabil Nurs. 1998;23:204-9.

6. Fekete C, et al. Development and validation of a self-report version of the Spinal Cord Independence Measure (SCIM III). Spinal Cord. 2013;51:40-47.

7. Silver J, Ljungberg I, Libin A, Groah S. Barriers for individuals with spinal cord injury returning to the community: a preliminary classification. Disabil Health J. 2012. https://doi.org/10.1016/j. dhjo.2012.03.005

8. Phillips CO, Wright SM, Kern DE, Singa RM, Shepperd S, Rubin HR. Comprehensive discharge planning with postdischarge support for older patients with congestive heart failure: a metaanalysis. JAMA. 2004;291:1358-67. https://doi.org/10.1001/ja ma.291.11.1358.

9. David B, Preen Belinda ES, et al. Effects of a multidisciplinary, post-discharge continuance of care intervention on quality of life, discharge satisfaction, and hospital length of stay: a randomized controlled trial. Int J Qual Health Care. 2005;17:43-51.

10. Shepperd S, McClaran J, Phillips CO, Lannin NA, Clemson LM, McCluskey A, et al. Discharge planning from hospital to home. Cochrane Database Syst Rev. 2010;CD000313.

11. Carrie HK, Yam, Eliza, Wong LY, et al. Framework and components for effective discharge planning system: a delphi methodology. BMC Health Serv Res. 2012;12:396.

12. Bjørnshave Noe B, Bjerrum M, Ange $S$. The beginning of a new life following traumatic spinal cord injury-patient's experiences one month post-discharge. Int J Phys Med Rehabil. 2015;3:1.

13. Yang HY, Sanford JA. Home and community environmental features, activity performance, and community participation among older adults with functional limitations. J Aging Res. 2012; 2012.

14. Carr JJ, Kendall MB, Amsters DI, Pershouse KJ, Kuipers P, Buettner $\mathrm{P}$, et al. Community participation for individuals with spinal cord injury living in Queensland, Australia. Spinal Cord. 2017;55:192-7.

15. Balbale SN, Lones KA, Hill JN, LaVela SL. Examining participation among persons with spinal cord injuries and disorders using photovoice. Qual Rep. 2017;22:1830-47.

16. Irgens I, Rekand T, Arora M, Liu N, Marshall R, BieringSørensen F, et al. Telehealth for people with spinal cord injury: a narrative review. Spinal Cord. 2018;56:643-55. 